Revue d'histoire de l'Amérique française

REVUE D.HISTOIRE DE L'AMÉRIQUE FRANÇAISE

\title{
Savoir-faire et changements techniques dans une entreprise d'ocre en Mauricie, 1892-1968
}

\section{François Lachance}

Volume 50, numéro 4, printemps 1997

URI : https://id.erudit.org/iderudit/305600ar

DOI : https://doi.org/10.7202/305600ar

Aller au sommaire du numéro

\section{Éditeur(s)}

Institut d'histoire de l'Amérique française

\section{ISSN}

0035-2357 (imprimé)

1492-1383 (numérique)

Découvrir la revue

\section{Citer cet article}

Lachance, F. (1997). Savoir-faire et changements techniques dans une entreprise d'ocre en Mauricie, 1892-1968. Revue d'histoire de l'Amérique française, 50(4), 511-535. https://doi.org/10.7202/305600ar
Résumé de l'article

Cet article tente de mettre en lumière les dynamismes d'une entreprise d'ocre en Mauricie dont les activités débutent à la fin du XIX ${ }^{\mathrm{e}}$ siècle et se maintiennent jusqu'à la fin des années 1960. L'ocre est un mineral utilisé en Europe depuis le milieu du XVIII ${ }^{\mathrm{e}}$ siècle comme pigment d'oxyde de fer dans l'industrie des peintures. L'attention se porte en particulier sur l'entreprise Canada Paint, qui produit des peintures à base d'ocre. Celle-ci, en plus de transformer considérablement le minerai sur place, fit appel à un artisan d'Angleterre spécialisé dans l'industrie des pigments. La place accordée à l'expérience et au savoir-faire d'un artisan jusque dans les années 1960 démontre la persistance d'un système technique qui s'enracine au milieu du $\mathrm{XVIII}^{\mathrm{e}}$ siècle et qui ne sera remplacé complètement qu'avec l'avènement des pigments synthétiques. 


\title{
SAVOIR-FAIRE ET CHANGEMENTS TECHNIQUES DANS UNE ENTREPRISE D'OCRE EN MAURICIE 1892-1968 1
}

\author{
FRANÇOIS LACHANCE \\ Centre interuniversitaire d'études québécoises \\ Université du Québec à Trois-Rivières
}

\section{RÉSUMÉ}

Cet article tente de mettre en lumière les dynamismes d'une entreprise d'ocre en Mauricie dont les activités débutent à la fin du XIX ${ }^{\mathrm{e}}$ siècle et se maintiennent jusqu'à la fin des années 1960. L'ocre est un minerai utilisé en Europe depuis le milieu du $\mathrm{XVIII}^{\mathrm{e}}$ siècle comme pigment d'oxyde de fer dans l'industrie des peintures. L'attention se porte en particulier sur l'entreprise Canada Paint, qui produit des peintures à base d'ocre. Celle-ci, en plus de transformer considérablement le minerai sur place, fit appel à un artisan d'Angleterre spécialisé dans l'industrie des pigments. La place accordée à l'expérience et au savoir-faire d'un artisan jusque dans les années 1960 démontre la persistance d'un système technique qui s'enracine au milieu du $\mathrm{XVIII}^{\mathrm{e}}$ siècle et qui ne sera remplacé complètement qu'avec l'avènement des pigments synthétiques.

\section{ABSTRACT}

This article highlights the dynamics of an ochre-producing business in the Mauricie region. The business began operation in the late $19^{\text {th }}$ century and lasted until the late 1960s. Ochre, a mineral, has been used in Europe since the middle of the $18^{\text {th }}$ century as a ferrous oxide pigment in the paint industry. Canada Paint, which produced ochre-based paints, did a fair amount of local transformation of the mineral and recruited an English artisan specialized in pigment-making. The importance given to artisan know-how and experience attests to the persistence of an $18^{\text {th }}$-centuryold technical system until the 1960s. This technical system was only replaced with the advent of synthetic pigments.

1. Cet article reprend une partie de mon mémoire de maîtrise, L'exploitation industrielle de l'ocre en Mauricie, Université du Québec à Trois-Rivières, 1995. Je remercie Claude Bellavance, René Hardy et Paul-Louis Martin pour leurs commentaires. 
Le changement technique tient depuis quelques années une place importante dans l'histoire des grandes organisations industrielles. Les questions qui dominent touchent le rapport entre le changement d'organisation des entreprises et l'innovation de même que le maintien d'un processus de renouvellement technique ${ }^{2}$. Le fait technique est surtout abordé d'un point de vue général et avant tout comme un déterminant de la performance des entreprises. Il en ressort un portrait impressionniste des procédés et façons de faire qui se sont succédé dans le temps.

Quelques contributions font heureusement exception et proposent un cadre d'analyse susceptible de saisir plus en profondeur les formes du changement ${ }^{3}$. Pour l'essentiel, ces apports considèrent le fait technique comme une suite d'actions coordonnées, des plus élémentaires aux plus complexes, organisée en un système interrelié et dépendant de systèmes voisins (culture, économie, législation, etc.). Retenons ici les apports de deux auteurs, Bertrand Gille et Thomas Hughes. Pour mettre en évidence l'interdépendance des procédés d'une période historique, Bertrand Gille propose la notion de système technique. Par exemple, trois systèmes se seraient succédé au XIX ${ }^{\mathrm{e}}$ siècle: classique (énergie hydraulique/bois), moderne (charbon/machine à vapeur/sidérurgie) et contemporain (électricité/moteur à explosion/chimie organique $)^{4}$. L'analyse de Thomas Hughes porte sur les traits particuliers de l'implantation de ces systèmes à l'échelle locale et sur leur grande variabilité. Cet auteur considère fondamentalement le fait technique non pas comme un fait caractérisé dans ses dimensions concrètes,

2. On consultera en particulier Giovanni Dosi, Renato Giannetti et Pier Angelo Toninelli, «Theory and History of Technology and Business Firms: the Microeconomics of Industrial Development», dans Giovanni Dosi, et al., dir., Technology and Enterprise in an Historical Perspective (Oxford, Clarendon Press, 1992), 1-26; Robert Boyer, «Cinquante ans de relations entre économistes et historiens: réflexions d'un économiste sur le cas de la France et des États-Unis», Annales ESC, 46,1 (janvier-février 1991): 67-101. Nous faisons également référence à l'ouvrage marquant d'Alfred D. Chandler Jr., Scale and Scope, the Dynamics of Industrial Capitalism (Cambridge (Mass.)/London (England), The Belknop Press/Harvard University Press, 1990). La traduction française, en trois tomes, est intitulée Organisation et performance des entreprises (Paris, Les Éditions d'Organisation, 1992).

3. C'est notamment le cas des œuvres majeures de Bertrand Gille et de Thomas Hughes, dont les travaux leur ont permis de définir les concepts de système technique pour l'un et de technological system pour l'autre. À ce propos, on doit consulter en particulier Bertrand Gille, dir., Histoire des techniques. Technique et civilisations. Technique et sciences (Paris, Gallimard, coll. «Encyclopédie de la Pléiade», 1978), 1652 p.; Thomas Hughes, «The Evolution of Large Technological Systems», dans Wiebe E. Bijker, Thomas P. Hughes et Trevor J. Pinch, dir., The Social Construction of Technological Systems. New Directions in the Sociology and History of Technology (Cambridge (Mass.)/London (England), The MIT Press, 1987), 51-82.

4. Alain Beltran et Pascal Griset, La croissance économique de la France 1815-1914 (Paris, Armand Colin, 1988), 92-93. 
mais comme un objet de construction sociale ${ }^{5}$. Somme toute, ces contributions nous invitent à inscrire la technique au sein de la globalité historique.

Au Québec, le changement technique n'est pas considéré comme un objet d'étude en soi. Quelques travaux ont intégré l'évolution technique à leur analyse, mais seulement pour mieux cerner $a$ priori les changements économiques et sociaux qui, de ce point de vue, en étaient les causes ${ }^{6}$. Notre démarche, au contraire, s'approche des perspectives d'analyse évoquées plus haut et a pour but de saisir les fondements de ce processus dynamique. De manière spécifique, cet article examine les conditions particulières d'exploitation et de réussite d'une entreprise d'ocre en Mauricie, d'abord sous l'impulsion d'investisseurs canadiens, puis d'une multinationale américaine. L'attention se porte sur les méthodes industrielles de l'exploitation de l'ocre, un pigment d'oxyde de fer utilisé notamment dans la fabrication des peintures. Nous analysons en particulier l'organisation technique des entreprises exploitantes.

L'absence d'archives de l'entreprise nous a amené à considérer sous un autre angle des sources généralement peu exploitées par les historiens de l'entreprise. En plus des monographies d'entreprises, des coupures de presse, des registres de déclarations sociales, des articles de revues spécialisées ainsi que l'ensemble de la documentation publiée, nous avons eu recours à des enquêtes orales, des relevés géologiques, des traités industriels contemporains et aussi des photogra-

5. Pour mettre en évidence le caractère global du développement technique, Thomas Hughes a émis plusieurs réflexions. Il propose la notion de technological style, qui résume l'essentiel de sa pensée: «Technological style can be defined as the technical characteristics that give a machine, process, device, or system a distinctive quality. Out of local conditions comes a technology influenced by time and place, a technology with a distinctive style. The local conditions external to the technology can be defined as cultural factors; the technology they shape, a cultural artifact. Among the cultural factors are geographical, economic, organizational, legislative, contingent historical, and entreprenarial conditions.» Thomas P. Hughes, Networks of Power. Electrification in Western Society, 1880-1930 (Baltimore, The Johns Hopkins University Press, 1983), 405. Cité par Yves Tremblay, «L'histoire des techniques comme champ historiographique», Jacques Mathieu, dir., Les dynamismes de la recherche au Québec (Québec, Presses de l'Université Laval, 1991), 243.

6. Yves Tremblay, loc. cit., 237-250. Comme le note l'auteur, l'histoire des techniques comme champ d'étude particulier reste en friche au Québec et au Canada. La contribution récente de René Hardy sur la sidérurgie québécoise au XIX ${ }^{\mathrm{e}}$ siècle fait cependant exception et démontre une connaissance approfondie des dimensions techniques de cette activité et de ses liens avec les aspects économiques et sociaux. Voir René Hardy, La sidérurgie dans le monde rural. Les hauts fourneaux du Québec au XIX siècle (Québec, Presses de l'Université Laval, 1995), 303 p. 
phies ${ }^{7}$. Nous avons aussi tiré profit de la correspondance de la famille Bradley, surintendants qui se succèdent de père en fils à la tête de l'établissement.

\section{L'ANCIENNETÉ DES PEINTURES D'OXYDE DE FER}

L'emploi des ocres de fer comme pigments à peinture est très ancien, comme l'attestent les peintures rupestres retrouvées par les archéologues dans plusieurs pays occidentaux, y compris au Québec et dans la région de la Mauricie ${ }^{8}$. Mais l'emploi de l'ocre n'est pas l'apanage des peuples sans écriture. Il se généralise aux époques suivantes. Ainsi, dans l'édition de 1884 du Nouveau manuel complet du fabricant de couleurs, l'introduction est consacrée aux «couleurs employées par les anciens ${ }^{9} »$. On y relève l'utilisation de l'ocre dès les premiers moments de l'Antiquité dans les fresques, bien sûr, mais aussi pour les édifices publics et les habitations de tout genre.

$\mathrm{Au} \mathrm{XIII}{ }^{\mathrm{e}}$ siècle, le traité du moine bénédictin Theophilus nous renseigne davantage sur les techniques de préparation des pigments de même que sur leur utilisation ${ }^{10}$. En plus de livrer maintes recettes de mélanges de pigments destinées à élaborer des peintures aux couleurs variées à base d'ocre (mais aussi de céruse, de gypse et d'autres ma-

7. Le premier corpus provient des rapports sur les entreprises minières publiés par les services gouvernementaux des mines, tant provincial que fédéral. On y trouve aussi les rapports annuels envoyés par les compagnies minières au gouvernement fédéral. Sans être exhaustifs, ces documents offrent tout de même de précieuses informations sur le volume et la valeur de la production des entreprises de même que le nombre de travailleurs engagés. Ils fournissent parfois une description sommaire de l'outillage et des procédés de fabrication utilisés. Des manuels techniques européens et américains des $\mathrm{XIX}^{\mathrm{e}}$ et $\mathrm{XX}^{\mathrm{e}}$ siècles traitant des technologies utilisées dans l'industrie des peintures sont aussi mis à contribution, de même qu'un fonds photographique renfermant près de 150 clichés portant sur des exploitations ocrières de la Mauricie. Ces photographies couvrent la période 1910-1950 et témoignent, pour la plupart, du site d'extraction et de transformation des ocres sur lequel porte cet article. Ensemble, elles offrent une vue presque complète de l'évolution des procédés de production et illustrent bien les diverses opérations du site minier: extraction, transport, affinage et expédition du minerai.

8. Dominique Zaban, «L'homme et la couleur», Jean Poirier, dir., Histoire des moeurs (Paris, Éditions Gallimard, 1990), 3 vols. Gilles Tassé et Selwyn Dewdney, Relevés et travaux récents sur l'art rupestre amérindien (Montréal, Université du Québec à Montréal, Laboratoire d'archéologie, coll. «Paléo-Québec», $\left.\mathrm{n}^{\circ} 8,1977\right), 122$ p. Gilles Tassé, Les peintures rupestres $d u$ lac Wapizagonke, Parc national de la Mauricie (Montréal, Université du Québec à Montréal, Laboratoire d'archéologie, 1976), $24 \mathrm{p}$.

9. Riffault, Vergnaud, Toussaint et Malepeyre, Nouveau manuel complet du fabricant de couleurs à l'huile et à l'eau, des laques, des couleurs fines, des couleurs hygiéniques, etc., contenant les meilleures formules, les procédés les plus nouveaux et les plus usités dans cette industrie (Paris, Librairie encyclopédique de Roret, 1884), $436 \mathrm{p}$.

10. Theophilus, On Divers Arts. The Foremost Medieval Treatise on Painting, Glassmaking and Metalwork (New York, Dover Publications, 1979), 216 p. Transcrit du latin, avec une introduction par John G. Hawthorne et Cyril Stanley Smith. 
tières premières), Theophilus révèle le traitement généralement accordé aux pigments avant leur utilisation. Le minerai ocreux fournit à l'état naturel une teinte jaune et, une fois brûlé, il devient rouge. Ensuite le coloriste broie lui-même l'ocre avec de l'huile de lin ou de l'eau. La technique est donc relativement simple et essentiellement artisanale. La préparation des peintures n'exige pas un procédé de transformation complexe ou l'intervention d'une main experte, mais uniquement la matière première, à peine transformée.

C'est à compter du XVIII ${ }^{e}$ siècle que débute en Europe l'exploitation du pigment minéral sous des formes industrielles. C'est le cas dans la région d'Auxerre, en France, dont les activités s'étendent de 1763 à 1966. Cette industrie est avant tout marquée par des pratiques artisanales. Essentiellement, l'utilisation d'une technologie rudimentaire et l'intervention d'un savoir-faire pratique caractérisent ce type d'exploitation à caractère familial ${ }^{11}$.

\section{LES PREMIERS ESSAIS INDUSTRIELS EN MAURICIE}

En Mauricie, la première tentative d'exploitation industrielle est faite au milieu du XIX $\mathrm{X}^{\mathrm{e}}$ siècle, à Pointe-du-Lac, par des entrepreneurs de New York ${ }^{12}$. Ils acquièrent les droits miniers de plusieurs lots d'agriculteurs et érigent un bâtiment d'exploitation ${ }^{13}$. Les installations sont rudimentaires et constituées principalement de fours pour calciner le minerai. L'ocre n'est donc qu'apprêtée sommairement, mise en barils puis expédiée. On se doute ainsi de l'importance de la part des coûts de transport de ces matières premières jusqu'à New York dans le prix de revient de l'entreprise. Celle-ci ne dure d'ailleurs pas plus d'une dizaine d'années. Le témoignage d'un contemporain à l'emploi

11. Jean-Charles Guillaume décrit ainsi les étapes du travail: d'abord, l'extraction à ciel ouvert permet de trier les ocres en deux catégories, l'ocre jaune commune et l'ocre fine. Après dessiccation du minerai sur des séchoirs laissés à l'air libre, l'ocre commune est pétrie avec de l'eau, moulée en pain et expédiée dans cet état au commerce sous le nom de «jaune commun". L'ocre fine, qui contient davantage d'oxyde de fer, est broyée «dans des bassins circulaires en pierre, à l'aide d'une meule verticale [...] puis passée au tamis et au bluteau à travers une soie très fine, clouée sur un cylindre pareil à ceux dont on se sert dans les moulins à farine». De l'ocre fine, on tire également l'ocre rouge qui s'obtient par «des procédés qui sont le secret des fabriquants». Avant le broyage, une sélection encore plus fine du minerai à l'extraction et son traitement thermique dans un four en briques sous un feu violent constituent les principaux éléments du procédé. Le savoir pratique du manufacturier détermine la réussite des opérations, en particulier lors de l'étape du traitement thermique. Jean-Charles Guillaume, Une industrie rurale: le travail de l'ocre dans l'Auxerrois 1763-1966 (Auxerre, Société des sciences historiques et naturelles de l'Yonne, 1991), $376 \mathrm{p}$.

12. Exploration géologique du Canada, Rapport de progrès pour l'année 1849-1850 (Toronto, Lovell et Gibson, 1851), 52.

13. Greffe du notaire Hubert Bellefeuille, actes $n^{\text {os }} 2327,2328,2329$ (30 juillet 1851) et $n^{\circ} 2338$ (23 août 1851), Archives nationales du Québec à Trois-Rivières. 
du gouvernement provincial pour promouvoir le développement industriel est d'ailleurs fort explicite: «Cette exploitation, dit-il, a été abandonnée à cause de la difficulté et du coût trop élevé du transport, ce qui n'existe plus depuis la construction du chemin de fer du Nord, qui passe pour ainsi dire au milieu de ces dépôts d'ocre ${ }^{14}$.»

Sur la rive nord de la région de Trois-Rivières, le réseau ferroviaire ne s'implante qu'à l'aube des années 1880 avec la création d'une première ligne connue sous le nom de Québec, Montréal, Ottawa et Occidental ${ }^{15}$. La mise en place du nouveau réseau de transport va susciter de nouvelles tentatives d'exploitation. De 1879 à 1893 , quatre entreprises tentent, encore en vain, de produire des pigments d'oxyde de fer pour la fabrication de peinture, cette fois à proximité d'un important gisement à Cap-de-la-Madeleine ${ }^{16}$. Pourtant, les entrepreneurs à la tête de ces sociétés parviennent aisément à financer leur entreprise $^{17}$. Ils profitent également de la proximité d'une ligne de chemin de fer. Mais, chaque fois, les industriels investissent si peu dans la transformation sur place que l'affinage des matières premières reste concentré dans la métropole montréalaise.

14. J.-C. Langelier, Le Nord ou esquisse sur la partie de la province de Québec située au nord du fleuve Saint-Laurent, entre l'Outaouais et le Labrador (Québec, I.-P. Dery, 1882), 134.

15. Gaétan Gervais, L'expansion du réseau ferroviaire québécois (1875-1895), thèse de doctorat (histoire), Université d'Ottawa, 1979, 538 p. Le phénomène se vérifie également en France, dans le canton d'Apt par exemple, où les débuts de l'exploitation de l'ocre sur une vaste échelle sont étroitement associés à l'arrivée du chemin de fer. La densité de la population et la proximité d'un marché important expliquent sans doute l'ancienneté de l'exploitation dont les vrais débuts remontent avant l'arrivée du chemin de fer, dans les dernières années de l'Ancien régime. Voir Pierre Simoni, L'industrie dans le canton d'Apt au XIXe siècle (Avignon, édité par l'Association pour la sauvegarde et la promotion du patrimoine industriel de Vaucluse, 1992), 130.

16. Il s'agit en 1879 d'une entreprise de Télesphore-Eusèbe Normand et des sociétés The Johnson Magnetic Iron Paint Company en 1888, William Johnson Company en 1889 et SaintMaurice Metallic Paint Company en 1891. Bureau d'enregistrement du comté de Champlain, Livre de renvoi officiel de la paroisse du Cap-de-la-Madeleine/Index aux immeubles; Joseph Obalski, Mines et minéraux de la province de Québec (Québec, Ministère des Mines, 1889), 35; «Geological and Natural History Survey», Mining Returns, Archives nationales du Canada; Registre des déclarations sociales, 1885-1895, Archives nationales du Canada.

17. À titre d'exemple, mentionnons l'industriel montréalais James Robertson qui constitue une importante entreprise métallurgique à Montréal, Toronto et Baltimore, et un commerce de métaux bruts, ainsi que le notable trifluvien Télesphore-Eusèbe Normand qui effectue des investissements dans de nombreuses affaires, notamment dans la construction de ponts, de chemins de fer et d'autres travaux publics. Voir François Guérard, Les notables de Trois-Rivières au dernier tiers $d u X I X^{e}$ siècle, Trois-Rivières, mémoire de maîtrise (études québécoises), Université du Québec à Trois-Rivières, 1984, 104-107; Jean Prince, Familles trifluviennes, notes généalogiques (Sillery, Éditions du Septentrion, 1989), 89; Peter Bischoff et Robert Tremblay, «James Robertson», Dictionnaire biographique du Canada, XII: 1891 à 1900 (Sainte-Foy, Presses de l'Université Laval, 1990): 981-982. 
L'entreprise Canada Paint change en profondeur cette façon de faire; l'impulsion donnée est nouvelle, tant sur le plan organisationnel que technique.

\section{CANADA PAINT (1892-1912)}

Dès son arrivée, la nouvelle entreprise Canada Paint ajoute des installations pour accroître la transformation du minerai sur place, procède à une réorganisation fonctionnelle du site industriel autour de son principal axe de développement, le chemin de fer, et, enfin, embauche un spécialiste pour diriger les opérations. La structure financière de Canada Paint se prête aussi plus facilement et plus adéquatement aux investissements dans le secteur minier que les entreprises qui la précèdent.

\section{Une organisation plus complexe}

Contrairement aux initiatives précédentes, où un seul investisseur dirigeait l'entreprise, Canada Paint adopte une structure financière qui permet de diminuer considérablement l'ampleur des risques pris par les investisseurs. Il s'agit, bien entendu, du principe de la société par actions. À sa fondation, la capitalisation de Canada Paint s'établit comme suit: 4500 parts à $100 \$$ chacune $^{18}$. Le principal bailleur de fonds, Samuel Findlay McKinnon, un investisseur torontois, siège à la direction de la compagnie à titre de président. On retrouve également six autres membres à la direction, tous actionnaires.

Entre 1892 et 1894, Canada Paint acquiert les droits d'exploitation des «terres à peinture» localisées sur dix lots appartenant à des agriculteurs du rang Saint-Malo, à Cap-de-la-Madeleine ${ }^{19}$. Contrairement aux pratiques des entreprises qui l'ont précédée, l'achat des droits miniers se transige selon un coût fixe qui varie de $300 \$$ à $400 \$$ par agriculteur et ce, à perpétuité. Canada Paint fait aussi l'acquisition des droits miniers de huit lots de l'une des premières entreprises d'ocre en plus de son établissement montréalais, le tout pour $250 \$$. Au total, la nouvelle compagnie investit $25000 \$$ pour lancer ses opérations. Les installations qui nécessitent le plus gros des investissements sont im-

18. B. T. A. Bell, The Canadian Mining Iron and Steel Manual. A Careful Digest of Information Relating to the History, Organization, and Operations of all Canadian Mining, Smelting and Iron and Steel Companies (Ottawa, 1894), 352. Canada Paint est enregistrée par lettres patentes le 23 janvier 1892 et incorporée à Montréal le 2 mars de la même année. Registre des déclarations sociales, 1885-1895, Archives nationales du Québec à Trois-Rivières.

19. Il s'agit en fait du second rang face au fleuve, à proximité d'une importante tourbière où l'on retrouve l'ocre. Bureau d'enregistrement du comté de Champlain, Livre de renvoi officiel de la paroisse du Cap-de-la-Madeleine/Index aux immeubles. 
plantées cette fois sur les lieux mêmes de l'extraction du minerai, à Cap-de-la-Madeleine. La fabrication des peintures nécessite en effet peu d'opérations après la calcination, le broyage et le tamisage du minerai. On incorpore simplement les pigments minéraux dans une solution composée le plus souvent d'huile de lin, d'un siccatif liquide et d'essence de térébenthine. C'est donc la transformation première du minerai qui requiert le plus d'équipements industriels ${ }^{20}$.

\section{L'aménagement du site}

Alors que les installations des premières entreprises se limitaient aux fours pour tirer du minerai grossier un pigment rouge à peine transformé, Canada Paint se dote dès le départ d'équipements plus perfectionnés. Afin de réduire les coûts de transport des pigments minéraux jusqu'à sa fabrique de Montréal, le minerai est davantage transformé sur place et réduit en fine poudre. Le chemin de fer assure le transport de la poudre d'ocre. Le rail est aussi mis à contribution pour acheminer plus efficacement le minerai d'un site d'opération à un autre.

L'extraction de l'ocre s'effectue à ciel ouvert. En dessous d'une mince couche de tourbe, le minerai est réparti inégalement à une profondeur qui va de 0,5 à 5,5 mètres dans des veines dispersées et il nécessite une cueillette sélective. Le travail se fait à main d'hommes, à l'aide de pioches et de pelles: on dépouille le sol des couches qui le composent en dégageant les strates, employant ainsi les mêmes méthodes d'extraction utilisées depuis plus de deux siècles aux Forges du Saint-Maurice ou encore plus tôt, en Europe ${ }^{21}$. Plutôt que de s'effectuer par charrette, comme auparavant, l'acheminement du minerai jusqu'aux ateliers de transformation met à profit un moyen de transport plus efficace, le rail, qui augmente considérablement les capacités de chargement. La traction chevaline demeure l'énergie motrice.

Après l'extraction, la transformation du minerai nécessite plusieurs opérations successives. On entasse d'abord une partie du minerai à proximité des bâtiments pour assurer ainsi le maintien des opérations durant la saison hivernale. On transporte par wagonnets l'autre partie du minerai dans un premier bâtiment abritant quatre fours de

20. Riffault, Vergnaud, Toussaint et Malepeyre, op. cit., $436 \mathrm{p}$.

21. André Bérubé, L'évolution des techniques sidérurgiques aux Forges du Saint-Maurice, 1: La préparation des matières premières (Québec, Parcs Canada, 1976), 121 p., Document inédit $n^{\circ} 221$. 


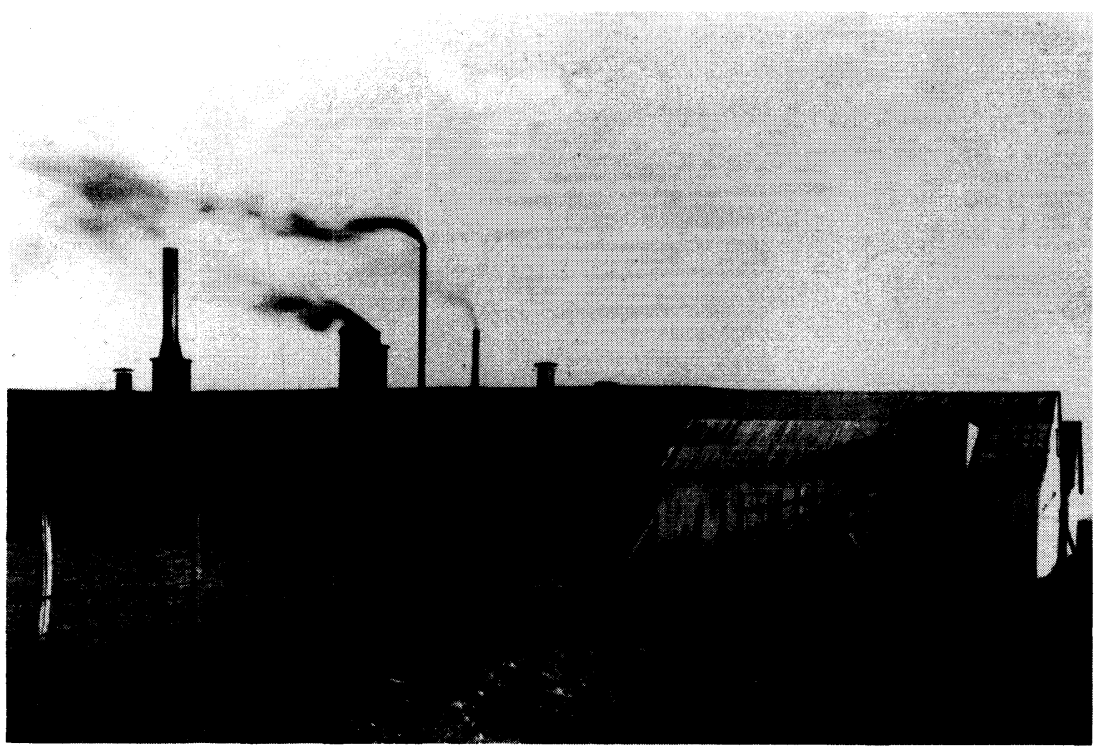

Le transport du minerai jusqu'au lieu de transformation par wagonnets sur rails, tirés par la force animale. CA 1908. Coll. privée James A. Bradley.

briques destinés à calciner l'ocre 22 . La calcination est le premier stade de la transformation du minerai ${ }^{23}$. C'est aussi l'étape cruciale de la préparation du pigment minéral: sous l'action de la chaleur, l'ocre jaune se transforme en ocre rouge ou en brun Van Dyck. Le fabricant de couleurs doit donc accorder beaucoup d'attention à cette opération afin d'obtenir l'éclat, la teinte et la couleur désirés. Après le traitement thermique, l'ocre est transportée dans un second bâtiment où se déroule une autre étape importante de la préparation des pigments d'oxyde de fer, le broyage.

On ne connaît pas dans les moindres détails les techniques employées pour broyer le minerai. En Europe, la pratique dominante demeure, encore au début du $\mathrm{XX}^{\mathrm{e}}$ siècle, l'emploi d'une meule analo-

22. Les fours sont construits de briques renforcées d'arêtes de fer. Ils comprennent une ouverture pour y introduire le minerai ainsi qu'un âtre à l'arrière pour le combustible, en l'occurrence, du bois. À l'extrémité des fourneaux, une cheminée permet d'évacuer la fumée qui se dégage lors du processus de calcination. La température à l'intérieur des fours varie de $800^{\circ} \mathrm{F}$ à $1200^{\circ} \mathrm{F}$. Entrevue réalisée auprès de James Bradley, 12 décembre 1991.

23. Le procédé de calcination permet de transformer l'oxyde ferrique hydraté en oxyde anhydre, en plus d'éliminer les matières organiques et végétales. 
gue à celles utilisées dans les meuneries ${ }^{24}$. Dans l'établissement de Canada Paint, il semble que les méthodes soient similaires ${ }^{25}$. Le broyage consiste à réduire l'ocre en poudre la plus fine afin d'obtenir une peinture à pigmentation résistante et de couleur uniforme ${ }^{26}$. Une machine à vapeur fournit la force motrice nécessaire au broyage. La dernière étape de l'affinage du minerai est celle du tamisage, qui permet d'obtenir un pigment uniforme d'une grande finesse. La poudre d'ocre est mise enfin dans des barils, fabriqués sur place par un tonnelier, et expédiée jusqu'à Montréal. On charge les barils d'ocre directement dans les wagons du Canadien Pacifique puisqu'une station ferroviaire a été construite sous le nom de Red Mill dès le début des activités de Canada Paint ${ }^{27}$.

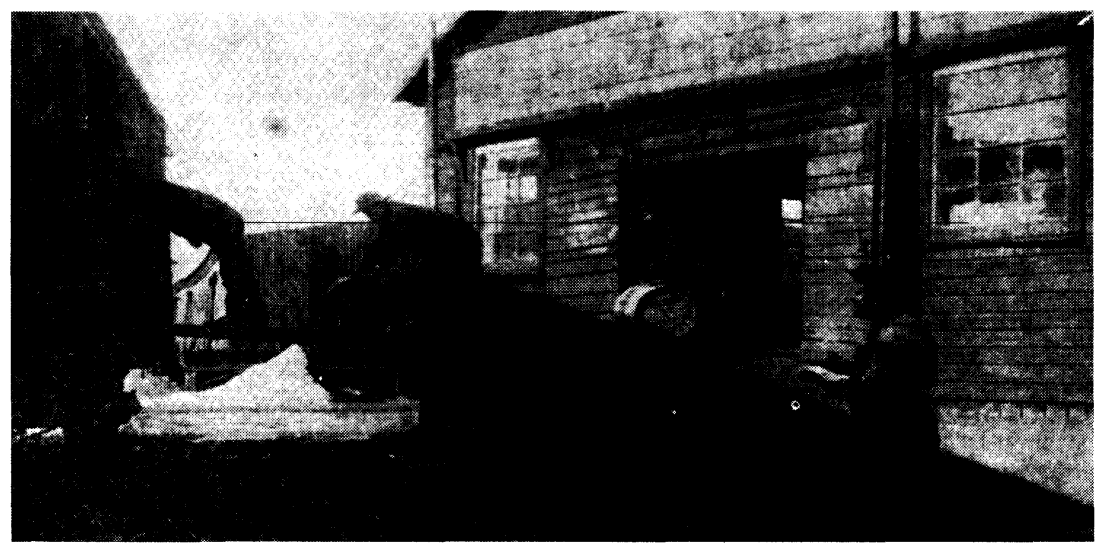

Le transbordement des barils emplis de poudre d'ocre dans les wagons du Canadien Pacifique. CA 1935. Coll. privée James A. Bradley.

24. C. Coffignier, Nouveau manuel du fabricant de couleurs (Paris, Librairie Bernard Tignol, 1926), 23; Jean-Charles Guillaume, op. cit., 78.

25. Joseph Bradley écrira en 1937: «The old method of grinding mineral pigments was by friction in the buhrstone type of mill, similar to the old-fashioned flour mill.» Voir Joseph Bradley, «Industrial Minerals Used in the Paint Industry», The Canadian Institute of Mining and Metallurgy, Transactions, 40 (1937): 390.

26. G. Champetier, H. Rabaté et J. L. Rabaté, Chimie des peintures, vernis et pigments (Paris, Dunod, 1956), 2: 373; C. Coffignier, "Couleurs et peintures», M. Matignon, dir., Encyclopédie de chimie industrielle (Paris, Librairie J.-B. Baillière et fils, 1924), 55-86.

27. Jean Roy, Daniel Robert et Louise Verreault-Roy, Les populations municipales et paroissiales de la Mauricie, 1850-1971 (Trois-Rivières, Publication du Groupe de Recherche sur la Mauricie, Université du Québec à Trois-Rivières, cahier n 3, 1980), 162. En collaboration, Itinéraire toponymique du Saint-Laurent: ses rives et ses îles (Québec, Études et recherches toponymiques 9,1984$), 108$. On désigne d'ailleurs la station ferroviaire sous le nom de «Red Mill»; le toponyme sera également attribué en 1915 au hameau du rang Saint-Malo lors de la création de la municipalité de Sainte-Marthe-du-Cap-de-la-Madeleine. 
Mais pour satisfaire aux normes de l'industrie de la peinture, ces méthodes conventionnelles de transformation de l'ocre requièrent aussi un savoir-faire relativement étendu.

\section{La part de l'expérience et du savoir-faire}

Avant même les débuts de la production, Canada Paint engage John Bradley, un ingénieur «spécialiste en couleurs» originaire de Derby, en Angleterre, et expérimenté, puisque déjà à l'emploi d'un manufacturier d'ocre ${ }^{28}$. Le recours à un spécialiste de l'industrie s'impose puisque l'art de tirer d'un minerai l'éclat, la teinte et la couleur désirés s'effectue durant le processus de calcination, en augmentant la température ou en prolongeant le temps de cuisson selon la composition du minerai extrait. Et c'est bien un art, au sens où la réussite de cette opération repose beaucoup plus sur l'acuité visuelle et l'expérience du spécialiste que sur une analyse chimique élaborée. À ce propos, le fils de John Bradley, Joseph, notera plus tard dans un article sur l'utilisation des pigments minéraux dans l'industrie de la peinture: «In all these calcining operations referred to, a certain technique has been acquired in the industry, through long experience with the various raw materials, to produce the ultimate in brilliance, tone, and tinting strength demanded by the trade ${ }^{29} . » C$ Ce savoir-faire technique contribue donc tout autant que les nouveaux équipements à répondre aux exigences de l'industrie.

Comparativement aux sociétés industrielles qui ont précédé $\mathrm{Ca}-$ nada Paint sur les sites miniers de la Mauricie à la fin du XIX ${ }^{\mathrm{e}}$ siècle, la réussite commerciale de cette dernière repose en bonne partie sur l'efficacité de son organisation technique. D'abord on complète les opérations qui doivent être menées sur le site d'extraction: ainsi l'ajout d'installations pour réduire plus finement le minerai en poudre permet de contrôler la qualité des pigments d'oxyde de fer expédiés, en plus de diminuer les coûts de transport. On confie ensuite l'opération la plus délicate - la calcination - aux mains d'un expert. Celuici a, de plus, la responsabilité de superviser le déroulement de l'ensemble des opérations. Finalement, on aménage l'usine et le site minier autour du rail, ajustant ainsi le rendement global des opérations à une technique efficace de transport.

28. Rosario Blanchet, «De Frontenac à nos jours, des Forges à Red Mill», Le Nouvelliste, 14 août 1945, 3. Enquête orale menée auprès de James A. Bradley, petit-fils de John Bradley et dernier surintendant du site.

29. Joseph Bradley, op. cit., 394. 
Dans l'ensemble, les techniques d'exploitation demeurent simples, mais doivent être coordonnées avec soin. Grâce à cette organisation technique, Canada Paint parvient à produire une quantité de pigments jamais égalée auparavant. En moyenne, l'usine produit près de 950 tonnes de minerai annuellement entre 1892 et 1911 , soit près du double de la quantité de minerai attribuée à chacune des entreprises précédentes $^{30}$. En outre, l'entreprise est en mesure de mieux contrôler la qualité des pigments expédiés à sa fabrique de Montréal, puisqu'ils sont ainsi prêts à entrer dans la coloration de la peinture. Le complexe de Red Mill est donc au cœur de cette filière technique, entre le prélèvement des matières premières et la distribution commerciale du produit fini.

L'arrivée sur la scène régionale de la société Sherwin-Williams conduit à l'intégration de l'exploitation des gisements d'ocre de la région dans le marché nord-américain des peintures.

\section{L'INTÉGRATION À UN GROUPE INDUSTRIEL AMÉRICAIN (1912-1929)}

$\mathrm{Au}$ début du $\mathrm{XX}^{\mathrm{e}}$ siècle, Sherwin-Williams tente de conquérir de nouveaux marchés ${ }^{31}$. La société acquiert alors plusieurs entreprises canadiennes: Martin-Senour Company Ltd, Lowe Brothers Company Ltd et Winnipeg Paint and Glass Company Ltd ${ }^{32}$. C'est pendant cette période d'acquisition qu'elle fait aussi l'achat, en 1912, du gisement exploité jusqu'alors par Canada Paint ${ }^{33}$. Afin de bien saisir les changements apportés par Sherwin-Williams au site de Red Mill, nous dressons d'abord un portrait général de l'entreprise, puis nous observons de plus près l'impact de l'arrivée en Mauricie de ce géant de l'industrie.

\section{Les caractères d'un "first mover»}

Sherwin-Williams est fondée en 1870 à Cleveland sous l'impulsion de trois entrepreneurs désireux de profiter de la croissance des

30. Sans compter que ces chiffres sont en-dessous de la réalité puisque le minerai est raffiné plutôt que seulement calciné. «Geological and Natural History Survey», Mining Returns, Archives nationales du Canada.

31. Alfred D. Chandler, Organisation et performance des entreprises, 1: Les USA 18801948 (Paris, Les Éditions d'Organisation, 1992), 262.

32. William Haynes, American Chemical Industry, 6: The Chemical Companies (Toronto/New York/London, D. Van Nostrand Company Inc., 1949): 385-390; C. J. S. Warrington et R. V. V. Nicholls, A History of Chemistry in Canada (Toronto, Sir Isaac Pitman and Sons Ltd, 1949), 337-339.

33. Bureau d'enregistrement du comté de Champlain, Livre de renvoi officiel de la paroisse de Champlain/Index aux immeubles. 
années de l'après-guerre civile américaine et de la forte demande qui l'accompagne ${ }^{34}$. Spécialisée au départ dans la fabrication et la vente de peintures et vernis, la nouvelle entreprise connaît une expansion rapide fondée sur plusieurs éléments: la mise en marché d'un produit de haute qualité grâce au développement d'un nouveau procédé qui permet de moudre plus finement les pigments, la constitution d'un large réseau de distribution et la vente d'un produit sous marque. L'entreprise engage, dans les années 1880, un chimiste formé au Massachusetts Institute of Technology ainsi que des spécialistes de la gestion. En investissant dans la recherche et dans la mise en place d'une organisation par départements spécialisés, et en favorisant la conquête de nouveaux marchés ainsi que la mise en marché de nouveaux produits, Sherwin-Williams se hisse rapidement parmi les leaders de l'industrie. Ses actifs atteindront un peu plus de 20 millions de dollars en 1917. Elle sera alors devenue le plus grand producteur de peintures et vernis au monde. Rien d'étonnant à ce qu'Alfred Chandler, dans son étude sur la grande entreprise industrielle moderne ${ }^{35}$, y voie les traits d'un first mover, soit une société qui effectue dans un secteur nouveau des investissements stratégiques autour des pôles organisationnels et techniques.

Le désir d'investir le marché canadien en distribuant un produit sous marque de même qualité que celui des États-Unis conduit Sherwin-Williams à remplacer en bonne partie les équipements de l'établissement de Canada Paint, tout en mettant à profit le savoir-faire du surintendant du site, John Bradley, ce salarié expert dans la préparation des pigments.

\section{Un nouveau complexe technique}

La transformation des opérations sur le site de Red Mill répond en fait à la mise en place d'un nouveau complexe technique qui, de surcroît, correspond à l'organisation de l'établissement autour d'une énergie nouvelle, l'électricité. Les processus de transformation de la matière première sont ainsi perfectionnés tandis que le produit (pigment) acquiert une valeur commerciale plus importante.

La localisation du minerai dans des terrains marécageux limite grandement la mécanisation des opérations d'extraction. Le travail se fait donc comme autrefois, à main d'hommes. Par contre, on change

34. Nous empruntons ici à Davis Dyer et Kathleen McDermott, America's Paint Company: a History of Sherwin-Williams (Cambridge, The Sherwin-Williams Company, 1991), $109 \mathrm{p}$.

35. Alfred D. Chandler, op. cit., 25-38. 
le mode de transport de l'ocre jusqu'aux ateliers de transformation en remplaçant la traction animale par la locomotion mécanisée.

Cependant, c'est surtout du côté des équipements de transformation que Sherwin-Williams apporte les plus importants changements à l'établissement de Red Mill. D'abord, on améliore sensiblement le rendement du procédé de calcination. Une étape, consistant à sécher l'ocre, s'ajoute au procédé de transformation du minerai ${ }^{36}$. De plus, le remplacement des fours en brique par des fours en fonte réduit la perte d'énergie tout en permettant d'atteindre une température plus élevée et plus stable ${ }^{37}$. Enfin, et surtout, on transforme complètement les équipements qui servent au broyage de l'ocre. En 1914, en effet, la nouvelle filiale de Sherwin-Williams signe un contrat de cinq ans avec l'entreprise d'électricité North Shore Power Company afin de mouvoir ses appareils à l'électricité ${ }^{38}$.

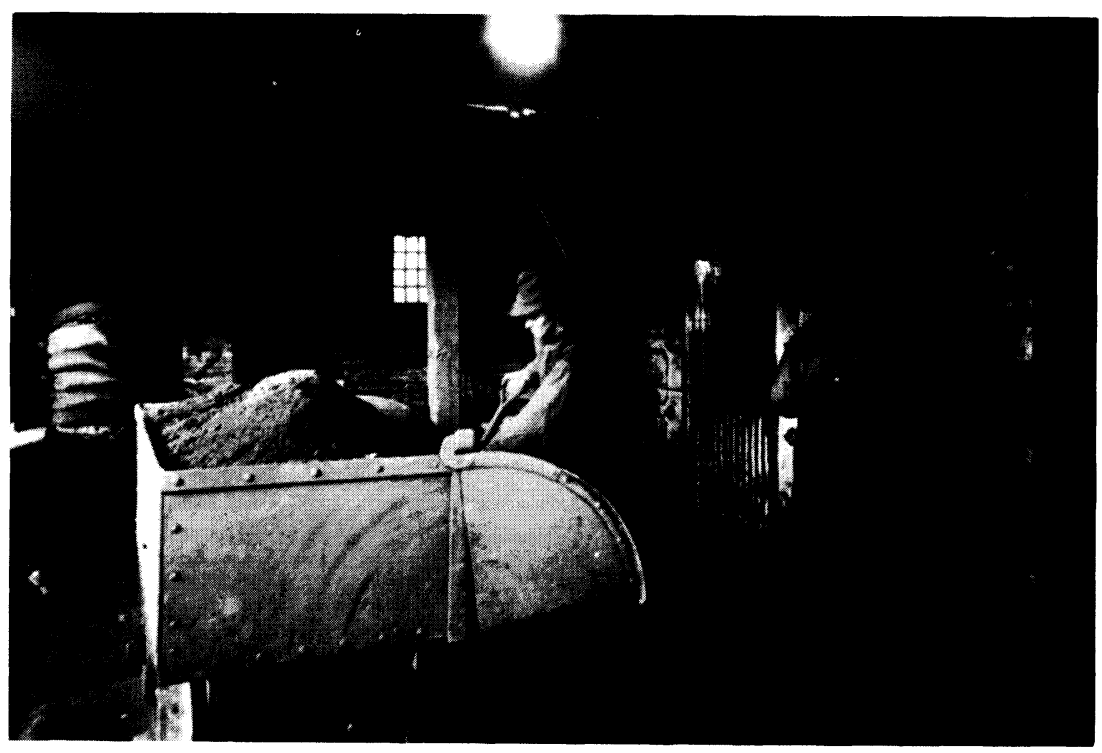

Le chargement de l'ocre dans les fours en fonte. CA 1935. Coll. privée James A. Bradley.

36. Ainsi avant la cuisson, «[...] the ore is dried in a Bartlett and Snow direct-heat counter-current rotary dryer, 4 feet in diameter and 40 feet long, revolving at 1,5 rpm.» Voir Joseph Bradley, «Industrial Minerals Used in the Paint Industry», The Canadian Institute of Mining and Metallurgy, Transactions, 40 (1937): 394.

37. Entrevue réalisée auprès de James A. Bradley, 12 décembre 1991.

38. Shawinigan Water and Power, Power Contracts Registers, 1914, 2 vol., F1A3/6 et F1A3/72, archives d'Hydro-Québec. 
La principale technique de transformation du minerai, à l'étape du broyage de l'ocre, repose sur le procédé nommé «air-flotation process», inventé aux États-Unis à la fin du $\mathrm{XIX}^{\mathrm{e}}$ siècle, mais appliqué dans l'industrie après $1910^{39}$. Le principe consiste à pulvériser le minerai dont les particules, maintenues en suspension à l'intérieur d'un cylindre tournant sur lui-même à grande vitesse, se fracassent alors entre elles. Il permet aussi de trier le minerai selon le volume des particules, à l'aide d'une série d'évacuateurs qui dirigent les plus grosses dans un broyeur mécanique, puis les redirigent dans le cylindre et ainsi de suite, jusqu'à ce que le minerai atteigne la finesse désirée. Ce procédé, à la fine pointe de la technologie de l'époque, permet d'obtenir un pigment d'une plus grande finesse, gage d'une peinture aux meilleures propriétés. À Red Mill, Sherwin-Williams implante ainsi le procédé:

The calcined ore is first passed through a scalping screen to remove tramp iron. This screen has the form of an enclosed revolving drum, sheated with perforated steel having 1/2-inch round holes. A magnetic pulley could not be used for this purpose, as a good proportion of the calcined ore is magnetic. From the scalping screen, the ore is fed to a Sturtevant swingsledge crusher with $1 / 8$-inch grates, this size providing an ideal mill-feed. The main grinding unit consists of three 42-in. Sturtevant rock emery mills, the discharge from which forms the feed for a 12 -foot Sturtevant classifier. The oversize from the air classifier is fed to a 30-in. vertical Sturtevant rock emery mill, the discharge from which returns into the classifier feed. The fines are led to a Mogul packer, and the finished product is packed in wooden barrels, holding 400 pounds each, and also in multi-wall paper bags holding 100 pounds each ${ }^{40}$.

Pour desservir avantageusement l'immense marché qu'elle couvre, la société Sherwin-Williams va aussi devoir s'assurer de la qualité constante des pigments produits.

\section{Contrôle de qualité et innovation}

Sherwin-Williams confie à l'établissement de Red Mill le soin de produire des pigments qui correspondent à ses critères de qualité de couleurs $^{41}$. Voici ce qu'en dit un ancien employé, affecté à cette tâche à partir de 1942 jusqu'au début des années 1960:

39. T. A. Rickard, A History of American Mining (New York, McGraw-Hill Book Company Inc., 1932), 397-414.

40. Joseph Bradley, op. cit., 394.

41. Devis et plan de la construction du laboratoire et de l'office à Red Mill, Fonds Caron, 1B01-2402.1B, Archives nationales du Québec à Trois-Rivières. 
Pendant la cuisson, j'allais au moulin chercher des échantillons à peu près toutes les heures. On prenait la peinture avec une espèce de gamelle qui était très longue, on la frottait sur un marbre pour définir les couleurs, à peu près [pendant] cinq minutes, puis après ça, on la mettait sur une vitre. Puis dans la vitre, on pouvait comparer si elle se rapprochait du standard qu'on voulait. Il y avait un seul standard de couleur, puis si on s'en approchait, ça voulait dire que la terre était bonne puis qu'elle était prête pour la mettre en poudre ${ }^{42}$.

Ce resserrement du contrôle de la production des pigments à Red Mill est exigé par le fait que l'établissement se retrouve intégré dans une entreprise au marché nord-américain ${ }^{43}$. L'établissement mauricien doit donc se conformer aux normes de qualité fixées par SherwinWilliams pour l'ensemble de son réseau de fabrication des peintures ${ }^{44}$.

La société américaine confie une autre responsabilité à son établissement de Red Mill, et en particulier au surintendant du site John Bradley, celle d'élaborer de nouveaux pigments. Rappelons qu'à la suite de l'acquisition de Canada Paint en 1912, Sherwin-Williams conserve John Bradley à la surintendance de l'usine de Red Mill ${ }^{45}$. Pour mettre à profit son savoir-faire, la multinationale dote l'établissement mauricien d'un petit laboratoire. C'est là que John Bradley, et plus tard son fils Joseph, vont produire de nouvelles couleurs avec l'ocre. Après une entrevue avec Joseph Bradley, un journaliste du quotidien Le Nouvelliste relate: «De l'oxyde de fer de Red Mill, on peut tirer trente-et-une couleurs différentes. [...] Vingt-sept couleurs de peinture ont été faites à Red Mill par feu John Bradley. Son fils, Joseph, en a découvert quatre autres ${ }^{46}$.» Ainsi, la multinationale américaine Sherwin-Williams, en tête des sociétés qui investissent dans la recherche, tire profit du savoir-faire de cet artisan spécialisé. De 1912 à 1965, trois générations de Bradley se succéderont à la direction de

42. Entrevue réalisée auprès de Robert Sauvageau, 25 octobre 1992.

43. C'est, du moins, ce qu'indiquent les rapports de l'entreprise envoyés au service fédéral des mines. "Geological and Natural History Survey», Mining Returns, Archives nationales du Canada.

44. La distribution des produits de l'entreprise Sherwin-Williams repose principalement sur l'effet de conditionnement des consommateurs, en associant les standards de qualité des produits de l'entreprise au nom «Sherwin-Williams». Dans cette veine, des guides pratiques de décoration sont proposés aux consommateurs et ainsi destinés à promouvoir les produits de l'entreprise. Plusieurs guides sont ainsi produits par Sherwin-Williams à partir de 1910 et ce, pendant une cinquantaine d'années. Consulter, par exemple, Your Home and its Decoration. A Series of Practical Suggestions for the Painting, Decorating, and Furnishing of the Home (Sherwin-Williams Company, Decorative Department, 1910), 204 p.

45. Entrevue réalisée auprès de James A. Bradley, 12 décembre 1991.

46. Rosario Blanchet, loc. cit. 


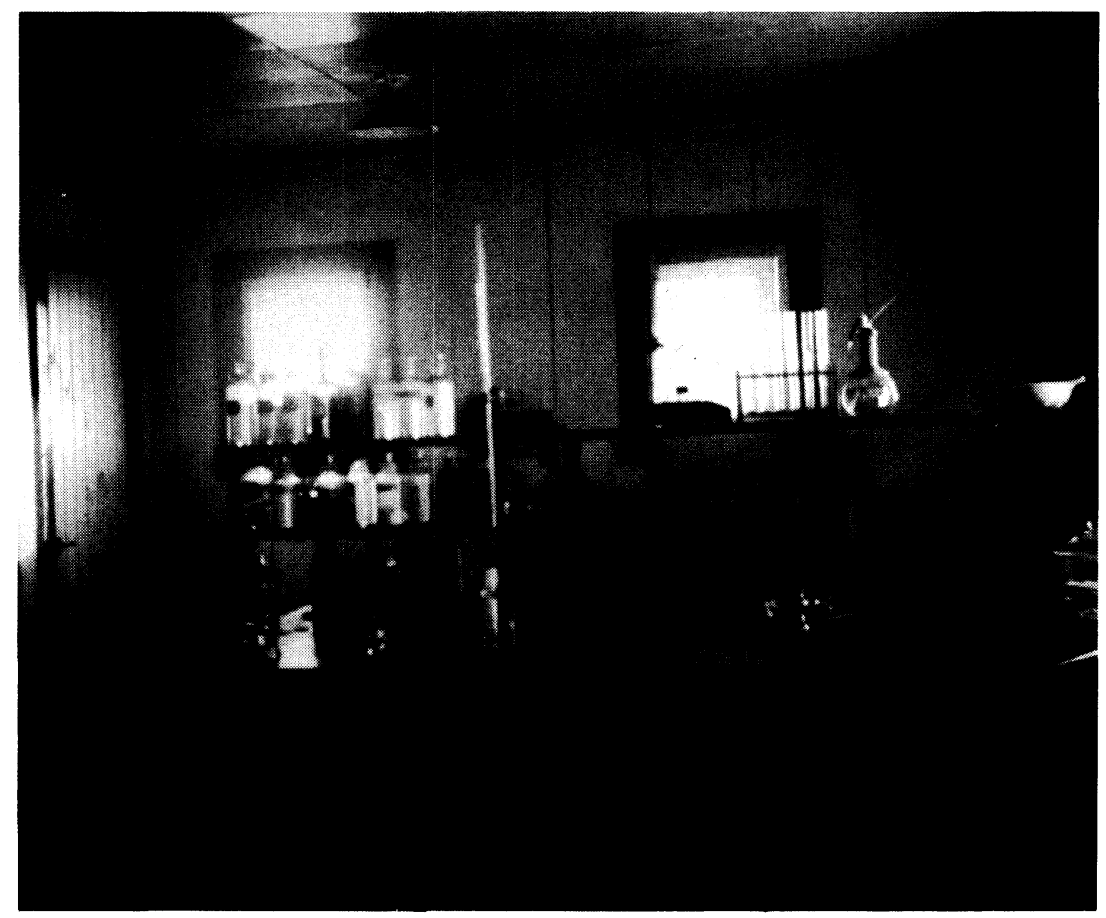

Le petit laboratoire. On peut y voir, en arrière-plan, des traités de sciences physiques et chimiques et à droite, à l'avant-plan, un lot de fioles contenant des additifs utilisés pour vérifier les propritétés des pigments. 1955. Coll. privée, James A. Bradley.

l'usine de Red Mill. Une véritable culture technique familiale est transmise d'une génération à l'autre, marquée à la fois par l'expérience pratique acquise dans l'entreprise et par un intérêt marqué pour les fondements scientifiques. Sherwin-Williams reconnaît l'étendue de la culture technique des membres de la famille Bradley et leur accorde plus que des fonctions de simples exécutants ${ }^{47}$. Ceux-ci jouent à l'échelle locale un rôle dynamique dans les innovations de la société américaine. Par ailleurs, Joseph Bradley se voit confier certains travaux de la Commission géologique du Canada sur les pigments minéraux $^{48}$.

47. La bibliothèque familiale, formée par trois générations de Bradley, est composée en grande partie d'ouvrages de sciences physiques et chimiques ainsi que de plusieurs traités industriels.

48. Deux des fils formés par Joseph Bradley à Red Mill, Gérald et Gordon, vont occuper des fonctions de superviseur dans un établissement industriel, l'un avec Aluminum Company en Guyane anglaise et l'autre, avec Wabasso Cotton. 
L'arrivée de Sherwin-Williams en Mauricie correspond à la mise en place d'un nouveau complexe technique, plus perfectionné mais surtout plus efficace. La production minière de l'usine de Red Mill augmente de $80 \%$ entre 1912 et $1919^{49}$. Puis, Sherwin-Williams accroît le contrôle de la production. Avec l'achat d'une seconde entreprise d'ocre en 1930, Champlain Oxyde Company, Sherwin-Williams devient le seul producteur de pigments en Mauricie. Cette activité est ainsi entièrement intégrée dans une organisation dont les dimensions n'ont plus rien de comparable avec Canada Paint. La production locale de pigments dépend désormais des stratégies d'une multinationale américaine.

La pénurie des matières colorantes qui sévit aux États-Unis à partir de la Première Guerre mondiale incite Sherwin-Williams à conquérir de nouveaux secteurs de l'industrie chimique, ce qu'elle fait notamment à partir de 1930, mettant à contribution son usine de Red Mill. Dans quelle mesure cette petite industrie rurale contribue-t-elle aux stratégies de diversification de produits adoptées par SherwinWilliams? Le savoir-faire de la famille Bradley intervient-il de façon significative dans l'atteinte de ces nouveaux objectifs? Autrement dit, dans quelle mesure cette culture familiale, scientifique et technique, fut-elle influente au sein de la filiale de la multinationale en Mauricie? Ce sont quelques-uns des aspects que nous allons maintenant examiner.

\section{LA DIVERSIFICATION ET LE DÉCLIN DES ACTIVITÉS (1930-1968)}

Au cours des années 1920 et 1930, Sherwin-Williams réorganise son entreprise autour de nouveaux secteurs et investit massivement dans le développement de produits synthétiques ${ }^{50}$. D'abord, l'élaboration de laques et de résines synthétiques à la fin des années 1920 permet à Sherwin-Williams de conquérir rapidement le secteur aéronautique aux États-Unis ainsi qu'une partie du secteur de l'industrie automobile. Pourtant, loin encore de distribuer massivement et exclusivement des peintures à base de pigments synthétiques, elle continue d'étendre ses activités en Amérique centrale et en Amérique du sud où elle fonde plusieurs établissements industriels et commerciaux. En fait, ce n'est qu'après la Deuxième Guerre mondiale que l'entreprise va véritablement parvenir à commercialiser ses produits synthétiques.

\footnotetext{
49. «Geological and Natural History Survey», Mining Returns, Archives nationales du Canada.

50. Davis Dyer et Kathleen McDermott, op. cit.
} 
Dans l'intervalle, Sherwin-Williams effectue des investissements dans d'autres gammes de produits, les insecticides par exemple. L'entreprise de Red Mill va d'abord profiter largement de cette période de transition. Elle réussira à maintenir sa position dans le marché canadien tout en réagissant particulièrement bien aux réorientations de la multinationale. La période de la Seconde Guerre va donner une impulsion nouvelle aux activités de Sherwin-Williams à Red Mill.

Jusqu'en 1939, l'usine continue de tirer son principal profit du marché des pigments à peinture. Cependant, les difficultés s'accumulent. Dès le début des années 1930, le superviseur de SherwinWilliams à Montréal se plaint de ce que les pigments de l'usine de Red Mill ne répondent plus aux standards de couleurs définis par la multinationale ${ }^{51}$. De nouveaux débouchés doivent être trouvés si l'on veut maintenir les activités de l'usine. En conservant les mêmes procédés de transformation, le minerai est utilisé, cette fois, comme matière colorante dans la fabrication du linoléum, du plastique, du béton, etc., comme agent nutritif (micro-élément) incorporé dans les fertilisants et comme abrasif pour le polissage des verres optiques et des bijoux. Plusieurs entreprises canadiennes profitent ainsi du faible coût du minerai transformé à Red Mill. La période de la Seconde Guerre provoque la rareté de certaines ressources comme les pigments minéraux et contribue à consolider cette demande nouvelle. Ainsi, la poudre d'ocre est utilisée comme abrasif pour le polissage des verres des projecteurs de la défense antiaérienne (DCA). S'y ajoutent bientôt la manufacture de pigments d'oxyde de zinc et la préparation de DDT. Ces deux activités nouvelles se greffent aux opérations de l'usine au même moment, mais résultent d'impulsions différentes: la première est due à l'esprit d'innovation du responsable local de SherwinWilliams et la seconde, aux investissements réalisés par la multinationale dans de nouveaux créneaux industriels.

Durant la Seconde Guerre, une pénurie d'oxyde de zinc suscite de nombreuses expérimentations dans le but de trouver un substitut à ce pigment utilisé pour la fabrication de peintures blanches. La multinationale confie alors au surintendant de sa filiale à Red Mill, Joseph Bradley, le soin de prendre les moyens nécessaires afin d'exploiter à titre d'essai un sous-produit d'oxyde de zinc $^{52}$. L'usine de Trail, en Colombie britannique, approvisionne l'entreprise de Red Mill en

51. Correspondance de la famille Bradley, 1925-1967, coll. privée, James A. Bradley.

52. Entrevue réalisée auprès de James A. Bradley, 12 décembre 1991; Rosario Blanchet, «Surintendants de père en fils: la famille Bradley à l'usine de Red Mill», Le Nouvelliste, 15 août $1945,3$. 
matières premières: les résidus provenant de la production de la matte cuivreuse de l'établissement sidérurgique composent en effet une substance grise sombre jusque-là inutilisée comme pigment ${ }^{53}$. Joseph Bradley va parvenir à mettre au point un procédé permettant de tirer profit de ces rejets.

Quelques tentatives faites au cours des années 1930 n'avaient donné aucun résultat concluant ${ }^{54}$. Le problème le plus fréquemment rencontré dans la fabrication des pigments minéraux se situe à l'étape du traitement thermique des matières premières. L'intensité et la répartition de la chaleur déterminent en partie la qualité des pigments produits. Or, il semble que les résidus d'oxyde de zinc soient particulièrement sensibles à la chaleur, puisque les fours conventionnels utilisés pour chauffer les autres minéraux ne sont pas efficaces ${ }^{55}$. Sous l'action de la chaleur, les résidus de zinc deviennent certes tous blancs, mais ils sont de teintes différentes. Pour solutionner ce problème, Joseph Bradley entreprend de mettre au point un nouveau four.

Le surintendant de l'usine de Red Mill reprend ses observations d'une expérience menée une dizaine d'années plus tôt pour produire des pigments synthétiques d'oxyde de fer à partir de sulfate de fer. Le sulfate de fer est un sous-produit de la transformation du minerai de fer en fonte, similaire au sous-produit d'oxyde de zinc. Mais l'importation de ce sous-produit des établissements sidérurgiques américains s'étant avérée trop couteuse ${ }^{56}$, l'expérimentation avait dû être rapidement abandonnée. Néanmoins, cette expérience va permettre d'ajouter aux installations l'outillage nécessaire au traitement thermique du zinc: un four rotatif qui permet de mieux répartir l'intensité de la chaleur.

Joseph Bradley se renseigne aussi sur les modèles de fours rotatifs et leur fonctionnement. Il consulte de nombreux brevets américains ainsi que plusieurs revues spécialisées telles Canadian Paint \& Varnish Magazine et Paint, Oil and Chemical Review, en plus d'entretenir des échanges fréquents avec les superviseurs de SherwinWilliams à Montréal et à Toronto ${ }^{57}$. Il en arrive ainsi à mettre au point un four rotatif capable de produire des pigments synthétiques d'oxyde de zinc qui sera utilisé pendant toutes les années de guerre.

53. Alfred W. G. Wilson, Industries métalliques du cuivre au Canada (Ottawa, Ministère des Mines, Imprimerie du Gouvernement, 1917), 83-100.

54. Rosario Blanchet, «De Frontenac à nos jours, des Forges à Red Mill», Le Nouvelliste, loc. cit.

55. Entrevue réalisée auprès de James A. Bradley, 12 décembre 1991.

56. Joseph Bradley, op. cit., 387.

57. Correspondance de la famille Bradley, 1925-1967, coll. privée, James A. Bradley. 
Durant cette période, une autre activité s'ajoute aux opérations de la filiale de Red Mill, la fabrication de DDT. Au cours des années 1940, en effet, les investissements de Sherwin-Williams dans de nouveaux secteurs atteignent des proportions gigantesques; l'entreprise devient même le plus grand producteur d'insecticides aux États-Unis, mettant en marché une large gamme d'insecticides, de fongicides et de désherbants ${ }^{58}$. Plusieurs établissements industriels de l'entreprise multiplient alors leurs activités. À sa filiale de Red Mill, SherwinWilliams manufacture un insecticide organique, le dichloro-diphényl trichloréthane (DDT).

La poudre d'oxyde de zinc produite à Red Mill est sans doute utilisée comme support au DDT. Cet insecticide organique de synthèse est en effet utilisé dans un mélange sous forme de poudre afin de permettre la dispersion du produit. C'est, du moins, l'hypothèse qui semble la plus plausible pour expliquer la production de cet insecticide à Red Mill. Rappelons que c'est à la fin des années 1930 que les propriétés insecticides du DDT sont d'abord mises en évidence. Les études de l'époque démontraient l'action spectaculaire du DDT sur les insectes et son innocuité sur l'homme. Durant la guerre, le produit est largement utilisé par les troupes alliées pour combattre la propagation des poux, du typhus et de la malaria. Au printemps de l'année 1943, chaque soldat allié reçoit un sachet de poudre de DDT ${ }^{59}$. SherwinWilliams tire profit de cette importante demande et met à contribution l'usine de Red Mill pour y répondre. L'entreprise bénéficie en plus de la disponibilité de la main-d'œuvre féminine à Red Mill durant la guerre pour empaqueter le DDT ${ }^{60}$.

À l'opposé de cette période d'intenses activités, les années 1950 et 1960 seront marquées par la décroissance.

\section{Les choix stratégiques de Sherwin-Williams (1950-1965)}

Sur plusieurs plans, la décennie des années 1950 annonce d'ores et déjà la fin de cette industrie de peinture en Mauricie. Essentiellement, le retour à l'économie civile et la restructuration imposent de nouveaux choix stratégiques qui laissent entrevoir, à moyen terme, la

58. Alfred D. Chandler, op. cit., 263.

59. Larry P. Pedigo, Entomology and Pest Management (New York/London, Macmillan Publishing Company/Collier Macmillan Publishers, 1989), 275-277. En 1948, P. Müller reçut le prix Nobel de médecine pour avoir découvert les propriétés insecticides du DDT. La publication, en 1962, de l'ouvrage de Rachel Carson, Silent Spring, sur les effets néfastes des pesticides sur l'équilibre de la chaîne alimentaire provoqua de vives réactions qui suscitèrent la formation du Presidential Science Advisory Committee on Environmental Quality.

60. Entrevue réalisée auprès de Robert Sauvageau, 25 octobre 1992. 
fin des opérations mauriciennes de la multinationale SherwinWilliams.

Dans les années 1950, la filiale de Sherwin-Williams doit de surcroît composer avec le problème croissant de la rareté des ressources. Robert Sauvageau, ancien assistant-chimiste à Red Mill, se remémore les soucis du surintendant James Bradley au cours de cette époque:

Jim [James A. Bradley] me disait, il va venir un temps où on ne sera plus capable de vendre notre produit. La mine était épuisée. On avait le minerai, mais il n'était pas uniforme, il ne se tenait pas. Les couleurs, une fois cuites, pouvaient faire 25 rouges différents. Les couleurs, eux autres [Sherwin Williams] quand ils disaient d'acheter une sorte, quand c'était raffiné, fini, il fallait que ce soit tout de la même couleur, du moins le plus rapproché. Puis je pense bien qu'avec toutes les terres que les mineurs ont levé partout, toute la meilleure était partie ${ }^{61}$.

Le fait était d'autant plus significatif qu'immédiatement après la guerre, la multinationale Sherwin-Williams avait cessé de diversifier ses établissements pour se concentrer à nouveau dans le secteur des peintures, fabriquées, cette fois, à partir de pigments synthétiques ${ }^{62}$. Dans les années 1950, 20\% de la population urbaine des États-Unis se déplace en milieu suburbain pour acquérir une maison neuve. Sherwin-Williams envahit littéralement le marché domestique grâce à la commercialisation des peintures synthétiques. À la fin de la décennie, ce marché représentera $80 \%$ des affaires de Sherwin-Williams ${ }^{63}$. Les pigments synthétiques permettent en effet de produire, à grande échelle, les mêmes standards de couleur ${ }^{64}$, tandis qu'à l'inverse, les pigments minéraux peuvent difficilement répondre à cette stratégie de distribution massive de produits parfaitement identiques. Il apparaît de plus en plus difficile pour Red Mill de maintenir ses opérations dans un tel contexte. Pressentant les effets de l'arrivée sur le marché de pigments synthétiques, James A. Bradley va tenter de trouver de nouvelles vocations pour l'établissement qu'il dirige au nom de SherwinWilliams.

Dès le début des années 1950, James A. Bradley entretient des échanges fréquents avec les ministères canadiens et américains, responsables des mines, dans l'espoir d'exploiter les résidus d'oxyde de fer produits dans plusieurs industries nord-américaines par la transformation de nombreux minerais comme le nickel, le plomb et la pyrite

61. Ibid.

62. Davis Dyer et Kathleen McDermott, op. cit., 60-71.

63. Ibid., 63 et 69 .

64. G. Champetier, H. Rabaté et J. L. Rabaté, op. cit. 
de fer. Ces démarches lui permettent même d'obtenir l'accord de principe de Canadian Johns Manville qui accepte d'approvisionner l'établissement de Red Mill en matières premières ${ }^{65}$. Mais ces efforts pour relancer l'entreprise ne trouvent pas écho chez les représentants de Sherwin-Williams.

En 1954, l'établissement industriel de Red Mill est fermé temporairement ${ }^{66}$. Le procès-verbal d'une rencontre organisée entre les représentants de Sherwin-Williams à Montréal et James A. Bradley nous informe de la solution adoptée: on réduit la masse salariale de l'entreprise de Red Mill de près de $48000 \$$, passant ainsi de 24 à 11 travailleurs, ainsi que la variété des pigments produits, dont le nombre passe de neuf à un seul ${ }^{67}$. Cette mesure permet de maintenir les opérations pendant 11 ans. Finalement, en 1966, Sherwin-Williams se retire et cesse ses activités à Red Mill. James A. Bradley tente alors, avec l'appui financier du gouvernement québécois, de relancer l'établissement industriel de Red Mill.

\section{Un dernier souffle}

Le 4 novembre 1966, Sherwin-Williams vend pour une somme symbolique l'ensemble des équipements ainsi qu'une quarantaine de lots miniers aux 12 employés de l'industrie, dont James A. Bradley qui prend la tête de la nouvelle société, Red Mill Industries Ltée ${ }^{68}$. Les activités reprennent un mois plus tard, grâce à une aide financière octroyée conjointement par le ministère de l'Industrie et du Commerce et la Banque de Montréal ${ }^{69}$. Le montant de l'aide financière s'élève à $25000 \$$, soit le coût des frais d'opération pour deux ans ${ }^{70}$. Au terme de ces deux années, l'entreprise Red Mill Indutries Ltée n'atteint cependant pas son seuil annuel de rentabilité, fixé à 800 tonnes de minerai ${ }^{71}$. Un rapport, envoyé en 1968 par le délégué du ministère de l'Industrie et du Commerce au gérant de la Banque de Montréal à Trois-Rivières, fournit les explications qui suivent:

65. Correspondance de la famille Bradley, 1925-1967, coll. privée, James A. Bradley.

66. «Fin de l'oxyde de fer: l'usine de Red Mill fermée dans 15 jours», Le Nouvelliste, 14 novembre $1954,1$.

67. Correspondance de la famille Bradley, 1925-1967, coll. privée, James A. Bradley.

68. Bureau d'enregistrement du comté de Champlain, Livre de renvoi officiel de la paroisse du Cap-de-la-Madeleine/Index aux immeubles.

69. «La Red Mill Industries Ltée reprend ses activités», Le Nouvelliste, 5 décembre 1966, 3.

70. Correspondance de la famille Bradley, 1925-1967, coll. privée, James A. Bradley.

71. Ibid. 
Comme Red Mill ne peut présentement produire que de l'oxyde de fer naturel, son marché est très réduit et ne pourrait justifier la continuation de l'exploitation par lui-même. J'ai donc essayé de vérifier s'il était possible d'obtenir un matériel qui pourrait permettre à Red Mill de produire des oxydes de fer synthétiques. [...] Durant mon voyage en Ontario, j'ai aussi appris qu'il existe un marché assez important pour de la barritine, un oxyde de baryum, employé par l'industrie de la peinture. Bien que ce matériel soit disponible au Canada à l'état brut [...], personne ne le traite ni ne le broye pour en obtenir la poudre très fine [...] requise pour les [...] manufacturiers canadiens de peinture. Donc, sans contredit, nous sommes en mesure de conclure que Red Mill Industries peut opérer d'une façon économique et profitable au sein des marchés qui existent au Canada, mais à deux conditions:

1 - Red Mill doit se procurer pour environ $55000 \$$ de machinerie nouvelle.

2 - Elle doit aussi trouver une source de financement lui permettant d'acquérir cette machinerie. Sans compromettre l'Office du Crédit Industriel du Québec, je crois qu'il serait possible d'en obtenir un prêt assez considérable mais [...] seulement lorsque de nouveaux capitaux de risque auront été investis ${ }^{72}$.

Aucun investisseur ne se présentera et l'entreprise Red Mill Industries Ltée déclarera faillite l'année même, mettant ainsi fin à un peu plus de 75 ans d'activités industrielles près du hameau de Red Mill, dans le comté de Champlain.

\section{CONCLUSION}

De toute évidence, on assiste progressivement à partir des années 1930 à la marginalisation de l'établissement de Red Mill face au géant américain et au nouveau système technique qu'il adopte. La production de guerre ne représente finalement qu'une occasion passagère dont profite l'usine de Red Mill, Sherwin-Williams mobilisant l'ensemble de ses établissements pour répondre à cette forte demande. Dans l'après-guerre, l'arrivée massive des produits synthétiques sur le marché annonce la fin de l'emploi des pigments minéraux dans la fabrication des peintures. Ce n'est désormais plus qu'une question de temps avant leur remplacement définitif.

On peut s'interroger sur les chances de survie de cette société si elle avait pu réunir les fonds nécessaires à sa restructuration. Le sa-

72. Ibid. 
voir-faire des Bradley n'était-il pas lié à l'ancienne façon de faire organisée autour de la fabrication des peintures à base de pigments minéraux? Pour y répondre, même en partie, on doit situer le problème dans une dimension plus large.

Ce qui ressort de la durée des activités à Red Mill, c'est la persistance, du moins à première vue, de techniques anciennes de fabrication des peintures jusqu'en plein cœur du $\mathrm{XX}^{\mathrm{e}}$ siècle alors que, depuis un bon moment déjà, les impératifs de production des entreprises sont orientés vers la distribution, à vaste échelle, de produits identiques. Cependant, Sherwin-Williams intègre à son organisation l'exploitation des gisements d'ocre de la Mauricie et renforce même la position de l'usine de Red Mill au sein de son réseau de fabrication des peintures. La société américaine renouvelle l'outillage et améliore les procédés, mais l'établissement de Red Mill demeure structuré autour de la production de pigments à partir des terres minérales. On est en présence d'un phénomène d'interpénétration de deux systèmes techniques. L'expérience et le savoir-faire d'un artisan continuent à jouer un rôle de premier plan dans la performance de l'établissement. À l'inverse, le rôle novateur des Bradley s'accroît au sein de la multinationale Sherwin-Williams. Il leur est confié la responsabilité de développer de nouveaux produits puis, durant la Seconde Guerre, celle d'innover dans les méthodes de fabrication des peintures blanches. L'introduction de la production de pigments synthétiques supplante graduellement l'ancien système technique organisé autour d'une technologie rudimentaire et d'un savoir-faire pratique dont l'origine remonte au $\mathrm{XVIII}^{\mathrm{e}}$ siècle en Europe. Au terme de ce changement technique, le savoir-faire des Bradley était devenu vétuste.

Cette étude nous a permis d'apprécier le rôle novateur d'un artisan spécialisé et d'évaluer l'importance accordée à son savoir-faire au sein d'une entreprise à la tête des sociétés qui investissent dans la recherche. Trop hâtivement peut-être, les savoir-faire, largement associés au monde artisanal à l'époque pré-industrielle, ont été mis en opposition avec le monde de la grande entreprise industrielle du $\mathrm{XX}^{\mathrm{e}}$ siècle. Cette conception s'oppose à notre vision de la dynamique du changement technique. L'expérience de Sherwin-Williams en Mauricie démontre que, au contraire, la multinationale a su intégrer avec profit ces savoirfaire dans son organisation et générer ainsi l'innovation dans son établissement régional. 